Duisburger Beiträge zur soziologischen Forschung 201503

\title{
Führungspositionen \\ im Ehrenamt - ein weiterer Bereich der Benachteiligung von Frauen?
}

Marcel Erlinghagen,

Belit Şaka und Ina Steffentorweihen
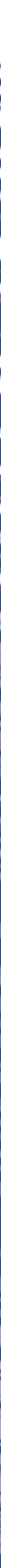

Institut für Soziologie | Universität Duisburg-Essen | Germany IfS 
Marcel Erlinghagen, Belit Şaka und Ina Steffentorweihen

Führungspositionen im Ehrenamt - ein weiterer Bereich der Benachteiligung von Frauen?

Duisburger Beiträge zur soziologischen Forschung 2015-03

doi: 10.6104/DBsF-2015-03

Herausgeber:

Institut für Soziologie, Universität Duisburg Essen

Institute of Sociology, University of Duisburg-Essen

https://www.uni-due.de/soziologie/

September 2015

Duisburger Beiträge zur soziologischen Forschung

ISSN 0949-8516 (Internet)

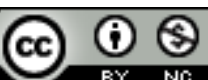

Creative Commons Lizenz CC BY-NC 4.0

Umschlagbild: Carmen Janiesch, Berlin

(C) 2015 by the author(s)

Marcel Erlinghagen ist Professor für Soziologie (Lehrstuhl Empirische Sozialstrukturanalyse) am Institut für Soziologie der Universität Duisburg-Essen.

Belit Şaka ist Wissenschaftliche Mitarbeiterin am Lehrstuhl für Empirische Sozialstrukturanalyse am Institut für Soziologie der Universität Duisburg-Essen

Ina Steffentorweihen ist Absolventin des Studiengangs MA Soziologie an der Universität Duisburg-Essen

marcel.erlinghagen@uni-due.de

belit.saka@uni-due.de

ina.steffentorweihen@stud.uni-due.de

\section{Downloads}

https://www.uni-due.de/soziologie/duisburger_beitraege/

\section{Redaktion}

Prof. Dr. Sigrid Quack

Institut für Soziologie

Universität Duisburg-Essen

Lotharstr. 65 | 47057 Duisburg | Germany

Tel. $+49203379-2259$ oder - 4794

Fax +49 203 379-5219

DBsF@uni-due.de 


\section{Abstract}

Based on data of the 2009 German Volunteer Survey ('Freiwilligensurvey') the paper analyses whether there is evidence for a discrimination of women in filling management positions in the volunteer sector. It turns out that women have a significant lower propensity to take up an unpaid volunteer management position. This correlation is also robust when we control for self-selection bias as well as certain important socio-demographic, socio-economic and volunteer-specific determinants. Further on we can show that this kind of gender-specific discrimination can be found particularly in leisure-time clubs and religious organisations. These results indicate that structural and organisational parameters play an important role to explain gender specific differences in filling volunteer management positions.

Keywords: volunteering, management positions, gender, discrimination, self-selection bias

\section{Zusammenfassung}

Der Beitrag geht mit Hilfe einer Analyse der Daten des Freiwilligensurveys 2009 der Frage nach, ob es im Bereich unbezahlter, ehrenamtlicher Arbeit Hinweise auf eine Benachteili-gung von Frauen bei der Besetzung von Führungspositionen gibt. Dabei zeigt sich, dass Frauen eine deutlich verringerte Wahrscheinlichkeit aufweisen, in ihrer ehrenamtlichen Tätigkeit Führungspositionen $\mathrm{zu}$ übernehmen. Dieser Zusammenhang ist auch dann stabil, wenn (Selbst-) Selektionseffekte sowie eine Reihe zentraler soziodemografischer, sozioökonomischer und engagementspezifischer Faktoren berücksichtigt werden. Eine differenzierte Analyse zeigt zudem eine Benachteiligung von Frauen hinsichtlich der Übernahme ehrenamtlicher Leitungsfunktionen insbesondere für bestimmte Tätigkeitsbereiche bzw. in bestimmten Organisationsformen. Vor allem Vereine und Kirchen bzw. religiöse Organisationen zeigen hier deutliche Defizite: Hier sind Frauen deutlich seltener als Männer mit ehrenamtlichen Leitungsaufgaben betraut. Dies sind wichtige Hinweise darauf, dass strukturelle und organisatorische Faktoren eine wesentliche Bedeutung für die Erklärung und mithin auch für eine zukünftige Beseitigung der auch im Bereich des Ehrenamtes vielfach existierenden „Gläsernen Decke“ zukommt.

Schlüsselwörter: Ehrenamt, Führungspositionen, Geschlecht, Benachteiligung, Selbstselektion 


\section{Inhaltsverzeichnis}

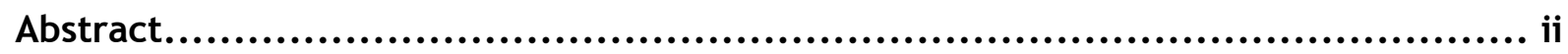

Zusammenfassung .............................................................. ii

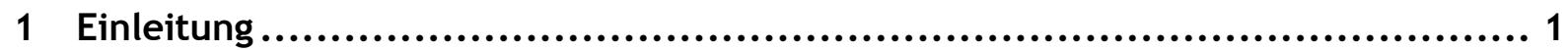

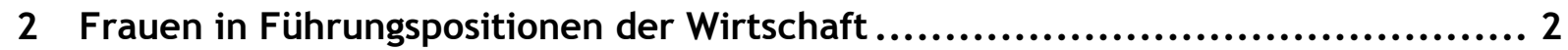

3 Frauen in ehrenamtlichen Führungspositionen .................................. 4

4 Daten und Methoden .................................................................. 7

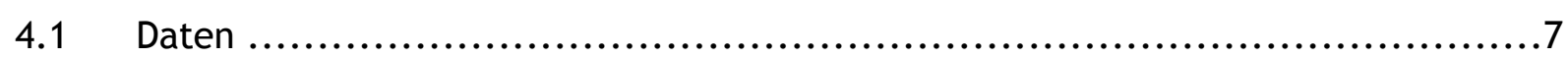

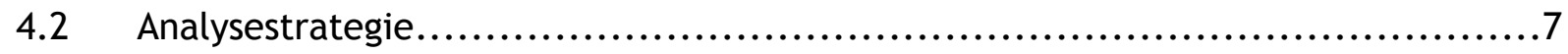

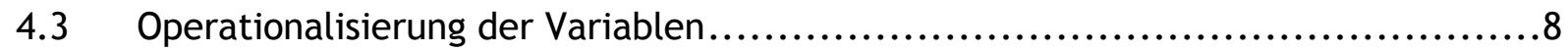

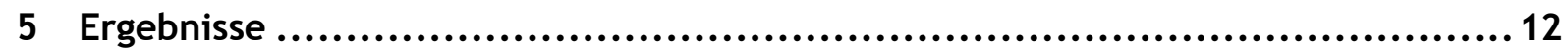

6 Zusammenfassung und Diskussion ................................................... 17 


\section{Abbildungsverzeichnis}

Abbildung 1: Average marginal effects für Frauen, eine Leitungsposition im genannten

Engagementbereich oder der jeweiligen Organisationsform zu bekleiden .. 16 


\section{Tabellenverzeichnis}

Tabelle 1: Deskription der Modellvariablen ......................................... 10

Tabelle 2: Verteilung der Frauen und Männer auf Ehrenämter und Leitungspositionen in Ehrenämtern ....................................................... 12

Tabelle 3: Leitungspositionen in Ehrenämtern, average marginal effects der Probit Regressionen mit und ohne Heckman-Selektion ........................ 13

Tabelle 4: Heckprobit-Modell - Erklärungsfaktoren der Leitungspositionen in Ehrenämtern ......................................................... 24

Tabelle 5: Kategorisierung der Engagementbereiche im Freiwilligensurvey 2009 ...... 26

Tabelle 6: Kategorisierung der Organisationsformen im Freiwilligensurvey 2009 ...... 26 


\section{Einleitung}

Auf dem deutschen Arbeitsmarkt sind Frauen nach wie vor in unterschiedlichen Dimensionen gegenüber ihren männlichen Kollegen benachteiligt. Dies zeigt sich zum Beispiel in der niedrigeren Bezahlung von Frauen, wobei dieser „Gender-Pay-Gap“ von derzeit rund 22 Prozent (Statistisches Bundesamt, 2014) in Deutschland im Vergleich zu anderen Industriestaaten besonders groß ist (OECD, 2013). Benachteiligungen zeigen sich auch darin, dass Frauen erheblich seltener Führungspositionen in Unternehmen bekleiden. Zwar wächst der Anteil weiblicher Führungskräfte, jedoch sind nach wie vor nur rund 25 Prozent der Führungspositionen in der Privatwirtschaft auf der obersten Ebene und knapp 40 Prozent auf der zweiten Ebene durch Frauen besetzt, wobei dieser Anteil mit zunehmender Unternehmensgröße deutlich abnimmt (Kohaut \& Möller, 2013; Holst, Busch, \& Kröger, 2012).

Die Ursachen für diese Benachteiligung am Arbeitsmarkt sind vielfältig (vgl. hierzu zusammenfassend und mit zahlreichen Literaturverweisen Bauernschuster \& Fichtl, 2013; Maier, Stettes, \& Queisser, 2013; Stettes, 2013). Bei näherer Betrachtung zeigt sich allerdings, dass viele dieser Ursachen wenn nicht unmittelbar so doch mittelbar auf unterschiedliche Rollenerwartungen zurückzuführen sind, die an Männer und Frauen gestellt werden. Häufigere Erwerbsunterbrechungen aufgrund familialer Verpflichtungen bei gleichzeitig inadäquaten institutionellen Kinderbetreuungsmöglichkeiten in Kombination mit geringeren Karriereaspirationen werden als mögliche Gründe dafür ins Feld geführt, weshalb sich Frauen in einem „Teufelskreis ökonomischer Rationalität“ (Ott, 1993) weniger stark als Männer auf Erwerbsarbeit spezialisieren. Entsprechend unterbleiben dann bestimmte Karriereschritte mit dem Effekt geringerer Entlohnung und mangelnder Aufstiegschancen. Auch die geschlechtsspezifische Segregation im Bildungs- und damit zusammenhängend Berufssystem (vgl. Reskin, 1994; Achatz, 2008b; Busch, 2013) wird als Ursache genannt. Beispielsweise wird argumentiert, dass mittlerweile zwar in etwa gleich viele Frauen und Männer ein Hochschulstudium absolvieren, Frauen jedoch in den naturwissenschaftlich-technischen Fächern deutlich unterrepräsentiert sind und daher die fachlichen Qualifikationen für eine Karriere in der Industrie und damit die Voraussetzung für gut bezahlte Führungspositionen in der Wirtschaft häufig nicht mitbrächten (vgl. exemplarisch BDA/BDI - Abteilung Arbeits- und Tarifrecht \& Abteilung Recht und Versicherung, 2015). Allerdings konnte in den vergangenen Jahren immer wieder gezeigt werden, dass die Benachteiligung von Frauen auch auf einer direkten Diskriminierung in Wirtschaft und Gesellschaft beruht und nur zum (kleineren) Teil auf inadäquate Qualifikationen und mangelnde Karriereorientierung zurückgeführt werden kann (vgl. exemplarisch Busch \& Holst, 2008; Jann, 2008; Hinz \& Auspurg, 2010).

Vor diesem Hintergrund geht der vorliegende Beitrag nun der Frage nach, ob es auch im Bereich unbezahlter, ehrenamtlicher Arbeit Hinweise auf eine Benachteiligung von Frauen gibt. Auf Basis der Daten des Freiwilligensurveys 2009 wird untersucht, ob Männer auch im Bereich des sozialen Engagements im Kontext von Non-Profit Organisationen (NPO) häufiger Führungspositionen einnehmen als Frauen oder aber, ob kein Unterschied zu beobachten ist bzw. im Bereich der unbezahlten, gemeinnützigen Arbeit 
möglicherweise sogar ein Vorteil für Frauen besteht, Leitungsfunktionen zu übernehmen. So könnte man einerseits erwarten, dass Frauen z.B. aufgrund einer höheren Affinität für Ausbildungen in sozialen Dienstleistungsberufen für Karrieren im Feld der gemeinnützigen Arbeit geradezu spiegelbildlich zur bezahlten Erwerbsarbeit Vorteile gegenüber potentiellen männlichen Konkurrenten besitzen müssten. Andererseits sind Führungspositionen auch im Non-Profit-Bereich mit Macht, Einfluss und Prestige verbunden, weshalb möglicherweise auch hier Männer aufgrund einer größeren Machtorientierung und Konkurrenzerfahrungen ähnlich wie im Erwerbsleben bevorteilt sind bzw. werden. Insofern trägt der vorliegende Beitrag nicht nur dazu bei, dass bislang in der nationalen und internationalen Forschung zu wenig beachtete Thema von Geschlechterungleichheiten im Bereich ehrenamtlichen Engagements stärker in den Fokus zu rücken. Von den Ergebnissen können auch mittelbar neue Erkenntnisse für die Debatte um die Benachteiligung von Frauen am Arbeitsmarkt gewonnen werden. Denn wenn sich zeigen sollte, dass Frauen auch in ihrer (scheinbaren) Domäne des sozialen Engagements bei der Besetzung von Führungspositionen benachteiligt wären, könnte dies das Argument entkräften, dass die mangelnde fachliche Passung eine wesentliche Hürde bei der Besetzung von Führungspositionen durch Frauen in der Wirtschaft darstellten.

Der Beitrag ist wie folgt aufgebaut: Kapitel 2 gibt zunächst einen komprimierten Überblick über den Stand der Forschung bezüglich Frauen in Führungspositionen in der deutschen Wirtschaft. Daran anknüpfend erörtert Kapitel 3, inwieweit die Ursachen der zu beobachtenden Benachteiligung bei der Besetzung von Führungspositionen in der Wirtschaft auch auf den gemeinnützigen und nicht gewinnorientierten Bereich ehrenamtlichen Engagements übertragen werden kann. Dabei werden die Gemeinsamkeiten und Unterschiede zwischen Erwerbsarbeit und unentgeltlicher ehrenamtlicher Arbeit herausgestellt und die im weiteren Verlauf empirisch zu überprüfende Fragestellung konkretisiert. Kapitel 4 stellt dann die Datenbasis und das methodische Vorgehen der vorgenommen Analysen vor, deren Ergebnisse in Kapitel 5 ausführlich dargestellt werden. Der Beitrag schließt mit einer Zusammenfassung und Diskussion der zentralen Befunde (Kapitel 6).

\section{Frauen in Führungspositionen der Wirtschaft}

Der Anteil von Frauen in den Vorständen der größten 200 Unternehmen (ohne Finanzsektor) lag in Deutschland im Jahr 2013 bei grade einmal 4,4 Prozent, der Anteil in Aufsichtsräten bei 15,1 Prozent (Holst \& Kirsch, 2014). Dagegen sind die Frauenanteile in Vorständen (12,6 Prozent) und Aufsichtsräten (18,1 Prozent) von Unternehmen mit Bundesbeteiligung (Holst \& Kirsch, 2014) und auch in der Führungsspitze im öffentlichen Sektor (Kohaut \& Möller, 2013) zwar deutlich höher, jedoch kann man auch hier nicht von ausgeglichenen Verhältnissen sprechen.

Die Bekleidung einer Führungsposition ist in der Regel abhängig von der Qualifikation, der (kontinuierlichen) Berufserfahrung und dem derzeit ausgeübten Beruf einer Person (Holst, 2008). Weitere Faktoren, wie Arbeitszeit bzw. Erwerbsstatus, Organisations- 
größe, Alter, Branche und Haushaltskontext, haben je nach Geschlecht allerdings unterschiedliche Auswirkungen auf die Wahrscheinlichkeit, eine Führungsposition zu bekleiden. So haben Frauen in kleineren Betrieben größere Chancen auf eine Führungsposition als in großen Unternehmen. Zudem wächst in Branchen mit einem höheren Frauenanteil die Wahrscheinlichkeit, dort auf Frauen in leitenden Funktionen zu treffen (vgl. Bechmann et al., 2013; Holst et al., 2012; Kohaut \& Möller, 2013; Krell, 2010). Anders als bei Männern korreliert die Übernahme einer Führungsposition darüber hinaus weniger mit steigendem Lebensalter bzw. wachsender Berufserfahrung (Kleinert, 2006). Zudem ist die Wahrscheinlichkeit für Frauen im potentiellen Familiengründungsalter zwischen 25 und 35 Jahren signifikant geringer, eine Führungsposition zu übernehmen als für Männer, unabhängig davon, ob Kinder tatsächlich vorhanden sind oder nicht (Holst, 2008; Krell, 2010). Und während sich für Männer Heirat und Familie positiv auf die Karriere auswirken, ist für Frauen eher das Gegenteil der Fall (Busch \& Holst, 2008). Ferner beschränken sich Frauen in Führungspositionen oftmals auf Bereiche, die nicht die direkte Führung des Betriebs betreffen. So findet man Frauen innerhalb von Führungspositionen vor allem in Bereichen des Personalwesens, der Personalentwicklung, der Bilanzbuchhaltung oder des Marketings (vgl. Kleinert, Kohaut, Brader, \& Lewerenz, 2007). Zudem haben Männer deutlich häufiger Positionen als leitende Vorgesetzte bzw. mit Weisungsbefugnis als Frauen (Busch \& Holst, 2008).

Die Geschlechter- und Organisationsforschung hat in den vergangenen Jahrzehnten unterschiedliche theoretische Erklärungsansätze entwickelt, die die Benachteiligung von Frauen am Arbeitsmarkt im Allgemeinen und die Unterrepräsentanz von Frauen in Führungspositionen im Besonderen erklären sollen (vgl. zu den folgenden Ausführungen insbesondere Müller, 1999; Achatz, 2008a, 2008b; Alemann, 2007; Ohlendieck, 2003):

(1) Eine wesentliche Ursache für die geringe Zahl weiblicher Führungskräfte insbesondere in Wirtschaftsunternehmen wird aus der beruflichen Segregation des Arbeitsmarktes abgeleitet. Aufgrund geschlechtsspezifischer Ausbildungsentscheidungen fehlten geeignete Bewerberinnen, da (immer noch) zu wenige Mädchen und junge Frauen mathematisch-naturwissenschaftliche bzw. ingenieurwissenschaftlich-technische Ausbildungsgänge („MINT-Fächer“) wählten, die bei vielen Unternehmen des produzierenden Gewerbes eine unabdingbare Einstiegsvoraussetzung darstellen.

(2) In diesem Zusammenhang wird zudem gerne auf generelle Differenzen zwischen Männern und Frauen hinsichtlich geschlechtsspezifischer Verhaltensweisen und Persönlichkeitseigenschaften verwiesen. So sind Männer karriereorientierter, zeigen einen aggressiveres Konkurrenzverhalten und einen hierarchischeren Führungs- und Kommunikationsstil und sind somit vielfach im Vorteil gegenüber potentiellen weiblichen Konkurrentinnen. Mittlerweile kann kaum mehr ernsthaft behauptet werden, dass eine solche Differenzierung (im Wesentlichen) biologisch begründet ist. Vielmehr ergeben sich klare Hinweise darauf, dass Verhaltensunterschiede zwischen Männern und Frauen zum größten Teil sozialisations- und kontextbedingt sind (vgl. dazu exemplarisch Penner 2008). 
(3) Ferner lässt sich die Benachteiligung von Frauen auch durch ideologische und strukturelle Barrieren erklären. Für organisationsinterne ebenso wie für unternehmensübergreifende Aufstiege relevante Netzwerke sind traditionell männlich dominiert und tendieren aufgrund von teils bewussten, teils unbewussten oder pfadabhängigen Schließungen dazu, Frauen von dieser wichtigen Ressource systematisch auszuschließen. In Kombination mit geschlechtsspezifischen Stereotypen und Vorurteilen führt dies zu unsichtbaren aber gleichwohl wirkungsstarken Hürden innerhalb von Unternehmen („gläserne Decke“), die für Frauen nur schwer zu überwinden sind. Erschwert wird dies zudem dann, wenn institutionelle Rahmenbedingungen die Arbeitsmarktchancen von Frauen zusätzlich verringern. Wenn Frauen die Rolle für Haushalt und Familie zugeschrieben wird und gleichzeitig die Möglichkeiten der institutionellen Betreuungsmöglichkeiten für Kinder oder aber pflegebedürftige Angehörige fehlen, sind dies denkbar schlechte Voraussetzungen, um mit einem männlichen Mitbewerber erfolgreich um eine vakante Führungsposition zu konkurrieren.

\section{Frauen in ehrenamtlichen Führungspositionen}

Legt man das sogenannte „Dritt-Personen-Kriterium“ zu Grunde, nachdem alle Tätigkeiten produktiv sind, bei denen prinzipiell eine Übernahme durch Dritte möglich wäre (also prinzipiell Marktfähigkeit vorliegt), so handelt es sich bei ehrenamtlichem Engagement eindeutig um eine bestimmte Form von Arbeit. Insofern eint Erwerbsarbeit und ehrenamtliche Arbeit, dass in beiden Fällen Güter oder Dienste in Anbindung an eine Organisation außerhalb des Haushalts (also z.B. Unternehmen oder Verein) produziert werden. Der wesentliche Unterschied besteht darin, dass definitionsgemäß Erwerbsarbeit gegen Bezahlung und ehrenamtliche Arbeit in der Regel unentgeltlich erfolgt. Hingegen erweisen sich Motive oder gar Inhalte als wenig geeignet, um ehrenamtliche Arbeit grundsätzlich von bezahlter Erwerbsarbeit zu unterscheiden (vgl. hierzu ausführlich Erlinghagen, 2000, 2003).

Ob und in welchem Ausmaß Menschen erwerbstätig sind, ist nicht nur wesentliche Voraussetzung für materiellen Wohlstand, sondern gleichzeitig auch wesentliche Vorbedingung für die Teilhabe am gesellschaftlichen Leben insgesamt. Dementsprechend ist die geringere Erwerbsbeteiligung und die geringere Entlohnung ein zentraler Indikator nicht nur für die Diskriminierung von Frauen am Arbeitsmarkt, sondern auch für ihre grundlegende gesellschaftliche Benachteiligung insgesamt. Arbeit erfüllt aber über das enge Motiv der Einkommenserzielung hinaus weitere Funktionen für Individuum und Gesellschaft. So hat Arbeit als produktive Tätigkeit für den arbeitenden Menschen einen sinnstiftenden Charakter, mit Arbeit ist oftmals die Zuschreibung von Prestige verbunden, Arbeitserfahrungen vergrößern das Wissen und die Kompetenzen des Arbeitenden und durch Arbeitskontakte kann wichtiges Sozialkapital aufgebaut werden; zudem hat Arbeit aus gesamtgesellschaftlicher Perspektive eine integrierende und stabilisierende Funktion (Promberger, 2008; Voß, 2010). Dies trifft gleichermaßen für bezahlte Erwerbsarbeit und unbezahlte ehrenamtliche Arbeit zu (Erlinghagen, 2003). 
Vor diesem Hintergrund wird auch deutlich, dass die Frage nach geschlechtsspezifischen Unterschieden hinsichtlich der Übernahme von Führungspositionen weit über den Aspekt der ökonomischen Benachteiligung von Frauen hinausgeht. Führungspositionen bedeuten in der Regel mehr Macht, mehr Gestaltungsmöglichkeiten und Autonomie, mehr Prestige, mehr Möglichkeiten sich weiter zu qualifizieren und wichtiges Sozialkapital aufzubauen. Diese Funktionen sollten gleichermaßen für bezahlte Führungspositionen in Unternehmen und unbezahlte (ehrenamtliche) Führungspositionen in gemeinnützigen Organisationen zutreffen.

Aus einer theoretischen Perspektive ist bislang offen, inwieweit die vorgestellten Erklärungen für eine Benachteiligung von Frauen am Arbeitsmarkt und ihre Unterrepräsentanz in Führungspositionen auch auf den ehrenamtlichen Bereich übertragbar sind. Die aus der beruflichen Segregation am Arbeitsmarkt abgeleitete Begründung scheint dabei kaum auf den Bereich des Ehrenamtes übertragbar. Im Gegenteil müsste man erwarten, dass aufgrund der spezifischen Ausbildungs- und Berufswahl von Frauen im sozialen Bereich sogar ein Vorteil von Frauen bei der Besetzung von Führungspositionen in gemeinnützigen Organisationen zu erwarten sein sollte; in eine ähnliche Richtung geht das Argument, mit ehrenamtlicher Arbeit stünde insbesondere Frauen eine Alternativrolle zur Verfügung, die als Ersatz für erfolgreiche Erwerbskarrieren fungieren könnte (Beck, 1999; Daniels, 1988, S. 122ff); Rotolo \& Wilson (2007) sprechen in diesem Zusammenhang vom „contrast effect“.

Differenztheoretische Überlegungen führen hingegen zu ambivalenten Hypothesen: Einerseits geht es bei Führungspositionen im Non-Profit-Bereich durchaus auch um Macht, Einfluss und Prestige, was dafür sprechen würde, dass aufgrund sozialisationsbedingter Verhaltensungleichheiten ${ }^{1}$ zwischen den Geschlechtern auch hier Männer wegen einer z.B. stärkeren Karriereorientierung und ihres ausgeprägten Dominanzverhaltens eher in Führungspositionen zu finden sein sollten. Rotolo \& Wilson (2007) verweisen in diesem Zusammenhang auf einen (negativen) „spill-over effect“ von Geschlechterrollen (vgl. hierzu auch Wilensky, 1961). Andererseits könnte aber der Umstand, dass in gemeinnützigen Organisationen gerade nicht die Profitmaximierung als Organisationsziel überwiegt, die Akzeptanz eines ,weiblichen', auf Konsens und Ausgleich beruhenden Führungsstils vergrößern. In diesem Fall könnten Frauen schließlich besonders gut für ehrenamtliche Führungsaufgaben geeignet sein und mithin müsste dann ein positiver Spill-Over-Effekt zu beobachten sein.

Hingegen sollten strukturelle und ideologische Barrieren für Frauen nicht auf den Arbeitsmarkt beschränkt bleiben. So kann man bspw. erwarten, dass auch in NPOs männliche Netzwerke die Etablierung von Frauen in Führungspositionen erschweren und dort ähnliche „gläserne Decken“ existieren wie in Wirtschaftsunternehmen. Und die Frage nach der Vereinbarkeit mit familiären Verpflichtungen stellt sich für Frauen eben nicht

\footnotetext{
1 Zur Diskussion um mögliche biologische Ursachen als Erklärung für geschlechtsspezifische Unterschiede bei ehrenamtlichen Aktivitäten vgl. Son \& Wilson (2010) und Wymer (2011).
} 
nur im Hinblick auf die Beteiligung an Erwerbsarbeit, sondern auch hinsichtlich der Beteiligung an ehrenamtlicher Arbeit, zumal dann, wenn mit einer Führungsposition in diesem Bereich mutmaßlich der zeitliche Aufwand für diese Tätigkeit zunehmen dürfte (Klenner, Pfahl, \& Seifert, 2001; Picot \& Gensicke, 2005).

Aus gender- und organisationstheoretischer Perspektive ist also zunächst offen, ob ähnliche Mechanismen, die im Erwerbsleben für die Benachteiligung von Frauen verantwortlich sind (negative Spill-Over-Effekte; „gläserne Decke“), auch im unbezahlten Ehrenamt wirken und dafür sorgen, dass auch hier Frauen seltener Führungsaufgaben übernehmen. Überraschenderweise sind Geschlechterunterschiede im Ehrenamt bislang von der Forschung ebenso wie von der Politik nur am Rande thematisiert worden (vgl. zu dieser Kritik Notz, 2006; Sing \& Hilpert, 2002). Auch in internationaler Perspektive liegen bislang vergleichsweise wenige Studien vor, die explizit geschlechtsspezifische Unterschiede im Zusammenhang mit ehrenamtlichem Engagement thematisieren. Wenn überhaupt werden diese Aspekte im Zusammenhang mit anderen zentralen Forschungsfragen eher beiläufig behandelt (für einen Literaturüberblick vgl. Helms \& McKenzie, 2014; Marshall \& Taniguchi, 2012; Rotolo \& Wilson, 2007).

Bezüglich der spezifischen Frage nach Frauen in ehrenamtlichen Führungspositionen deuten die wenigen vorliegenden Befunde darauf hin, dass Frauen auch im Ehrenamt seltener eine Führungsposition bekleiden als Männer. Während 39 Prozent der Männer in einer solchen Position sind, gilt dies nur für 24 Prozent der Frauen (Bundesministerium für Familie, Senioren, Frauen und Jugend, 2010). Männer sind zudem nicht nur insgesamt häufiger in Leitungs- und Vorstandspositionen zu finden, sie sind es auch dort, wo der Großteil der Ehrenamtlichen weiblich ist (vgl. Picot \& Gensicke, 2005; Zierau, 2001). Gleichwohl sind dies ausschließlich deskriptive Befunde, die zwar einen strukturellen Unterschied zwischen den Geschlechtern nachweisen, die jedoch keinerlei Rückschlüsse hinsichtlich der Ursachen für die Benachteiligung von Frauen bei der Besetzung ehrenamtlicher Führungspositionen erlauben. Es fehlen anspruchsvollere multivariate Analysen, die den Zusammenhang zwischen Geschlecht und ehrenamtlichen Führungspositionen unter Kontrolle wesentlicher weiterer Merkmale wie der Qualifikation, dem Alter oder aber dem Engagementbereich untersuchen (vgl. dazu Rotolo \& Wilson, 2007). Solche Analysen sollten dann auch eine mögliche Selektionsproblematik berücksichtigen, denn es könnte sein, dass die Unterrepräsentanz von Frauen in Leitungsfunktionen möglicherweise mit der zumindest in Deutschland zu beobachtenden generell niedrigeren ehrenamtlichen Beteiligung von Frauen (Erlinghagen, 2013) einhergeht. Die folgenden Analysen sollen dazu beitragen, diese Forschungslücke zu schließen. Wir gehen dabei der Frage nach, ob Frauen auch unter Kontrolle wesentlicher individueller und organisationsbezogener Faktoren und unter Berücksichtigung möglicher Selektionseffekte bei der Besetzung von ehrenamtlichen Führungspositionen benachteiligt sind. 


\section{Daten und Methoden}

\subsection{Daten}

Die Datengrundlage der Analyse bildet die dritte und damit derzeit aktuellste Welle des Freiwilligensurveys aus dem Jahr 2009. Der Freiwilligensurvey wurde 1999 von der Bundesregierung als repräsentatives Instrument zur Messung freiwilligen bzw. bürgerschaftlichen Engagements in der Bundesrepublik eingeführt, mit dem Ziel auf regionaler Ebene sowie Landes- und Bundesebene detaillierte und verallgemeinerbare Informationen zum Thema Ehrenamt zu sammeln (Gensicke, 2011). Die Befragung wird mit einem Abstand von vier Jahren wiederholt. Dabei werden nicht nur Umfang, Tätigkeiten und Bereiche des Engagements erhoben, sondern auch Motive für ehrenamtliches Engagement, Wünsche in Bezug auf das Engagement, die Engagementbereitschaft nicht engagierter Personen sowie Gründe für die Beendigung der Aktivität ehemals Engagierter. Darüber hinaus werden auch soziodemographische und sozio-ökonomische Merkmale, wie Geschlecht, Alter, Erwerbsstatus, Bildungsniveau, aber auch Informationen zum Zeitbudget einer Person und nicht zuletzt Informationen zu Leitungspositionen in Ehrenämtern abgefragt (Gensicke, 2011).

\subsection{Analysestrategie}

Der vorliegende Beitrag beschäftigt sich mit zwei Fragen: Erstens ist von Interesse, ob geschlechtsspezifische Unterschiede bei der Besetzung von Führungspositionen in Ehrenämtern zu beobachten sind. Zweitens soll daran anschließend überprüft werden, ob eine mögliche Benachteiligung von Frauen in bestimmten Tätigkeitsbereichen oder aber Organisationsformen (Verein, Kirche, Partei usw.) stärker oder schwächer ausgeprägt ist. Hierbei ist jedoch zu berücksichtigen, dass nur ehrenamtlich Engagierte auch eine unbezahlte ehrenamtliche Führungsposition in einer NPO übernehmen können. Das bedeutet, dass bei der Analyse, welche Determinanten das Bekleiden einer ehrenamtlichen Leitungsfunktion im Allgemeinen begünstigen und inwieweit es in diesem Zusammenhang zu Unterschieden zwischen Männern und Frauen kommt, diese Selektivität berücksichtigt werden muss (Sample Selection Bias). Auch bei der Frage nach den Unterschieden in den verschiedenen Bereichen oder Organisationsformen sind solche Selektionseffekte denkbar. Klassische Regressionsmodelle, berücksichtigen diesen Selektionsprozess nicht und würden in diesem Fall zu verzerrten Schätzungen führen (Heckman, 1979; Puhani, 2000). Um dieses Problem zu umgehen, werden im Folgenden sogenannte Heckman-Probit Modelle (Heckprob) geschätzt, die für Sample Selection Bias kontrollieren und anders als die klassischen Heckman-Modelle auf binäre abhängige Variablen angewendet werden können (Arminger, 1996; Wooldridge, 2002, S. 56off). Das besondere an Heckman Probit Modellen ist, dass zwei Gleichungen geschätzt werden; eine „Hauptgleichung“ und eine „Auswahlgleichung“. Für beide Gleichungen werden separate Vektoren der erklärenden Variablen zugelassen und die Korrelation der Fehlerterme (rho) der Hauptgleichung und der Auswahlgleichung geschätzt (Windzio, 2013, S. 270). Eine positiv oder negativ signifikante Korrelation beider Fehlerterme ist 
ein Hinweis darauf, dass diese zum Teil durch gemeinsame unbeobachtete Faktoren bedingt sind. Heckman Probit Modelle nehmen diese Korrelation der Fehlerterme mit in die Schätzung auf, um diese unbeobachtete Heterogenität und damit letztlich die Selektivität der Stichprobe zu kontrollieren.

In unserem Fall stellt die Belegung einer Führungsposition die abhängige Variable der Hauptgleichung dar. Die abhängige Variable der Auswahlgleichung ist ebenfalls eine dichotome Variable und nimmt den Wert Eins an, wenn die Person ehrenamtlich engagiert ist (sonst Null). In einem ersten Schritt (Auswahlgleichung) werden individuelle Wahrscheinlichkeiten berechnet, überhaupt ehrenamtlich aktiv zu sein. In einem zweiten Schritt wird dann (in der eigentlichen Hauptgleichung) die Wahrscheinlichkeit geschätzt, ob eine Person eine ehrenamtliche Führungsposition bekleidet, dabei jedoch die in der Auswahlgleichung identifizierte Selektivität berücksichtigt. In beiden Gleichungen wird für eine Reihe von unabhängigen Variablen kontrolliert, die zwar eine große Schnittmenge aufweisen. Dennoch gehen in die Hauptgleichung auch Prädiktoren ein, die nicht in der Selektionsgleichung vertreten sind und umgekehrt. In ähnlicher Weise werden auch die Schätzungen für Männer und Frauen vorgenommen. Schließlich werden dann weitere Selektionsmodelle geschätzt, die die zwischen ehrenamtlich tätigen Männern und Frauen mutmaßlich bestehenden unterschiedlichen Präferenzen für bestimmte Engagementbereiche bzw. Organisations-formen berücksichtigen. Dadurch wird es möglich zu prüfen, ob und inwieweit es geschlechtsspezifische Unterschiede bei der Bekleidung ehrenamtlicher Führungspositionen im Vergleich der unterschiedlichen Engagemetbereiche und Organisationsformen gibt.

\subsection{Operationalisierung der Variablen}

Um mögliche vorgelagerte Selektionseffekte zu berücksichtigen, werden zunächst Aktive sowie Nicht-Aktive in die Analyse aufgenommen. Ob Personen überhaupt ehrenamtlich aktiv sind, wird darüber erfasst, inwieweit die befragte Person zum Zeitpunkt der Befragung ein Ehrenamt ausübt ( $1=$ ja, $\mathrm{O}=$ nein) (zur aufwändigen und umfangreichen Erfassung ehrenamtlicher Aktivitäten im Freiwilligensurvey 2009 vgl. BMFSFJ, 2010, S. 91ff). Im Freiwilligensurvey wird das Bekleiden einer ehrenamtlichen Führungsposition über die direkte Frage abgebildet, ob der Befragte im Rahmen seines ehrenamtlichen Engagements eine Leitungs- oder Vorstandsposition hat. Daraus konstruieren wir die zentrale abhängige Variable, die den Wert 1 annimmt, wenn die Frage mit „Ja“ beantwortet wurde (ansonsten o). Liegen in einer der beiden abhängigen Variablen (ehrenamtliches Engagement, ehrenamtliche Leitungsfunktion) keine Informationen vor, werden diese Personen in der Analyse nicht weiter berücksichtigt. Insgesamt besteht der Datensatz aus 19.995 Personen, davon bekleiden 35 Prozent $(\mathrm{N}=6.996)$ Ehrenämter und von diesen haben wiederum 33 Prozent $(n=2.286)$ eine ehrenamtliche Vorstands- oder Führungsposition inne.

Neben der für unsere Analyse zentralen Unterscheidung zwischen Männern und Frauen wurden eine Reihe weiterer Variablen bei den Schätzungen berücksichtigt. So wird so- 
wohl in der Auswahl- als auch in der Hauptgleichung neben dem Alter, für den Migrationshintergrund, das Vorhandensein zu betreuuender Kinder im Haushalt, den Erwerbsstatus, den höchsten erreichten Schulabschluss und die regionale Herkunft (Ost-/Westdeutschland) kontrolliert.

Zusätzlich beinhaltet die Auswahlgleichung Prädiktoren, die das ehrenamtliche Engagement zwar bedingen, die aber aus theoretischer Perspektive keinen nennenswerten Einfluss auf die eigentliche Forschungsfrage haben sollten. Ergänzend zur Religiosität und dem Interesse an gesellschaftlichen und politischen Themen wird die Selbsteinschätzung der finanziellen Situation als Indikator für ökonomische Ressourcen, die Größe des Bekannten- und Freundesnetzwerks als Indikator für soziale Ressourcen und die Wohndauer im Ort als Indikator für Ortsverbundenheit in die Auswahlgleichung aufgenommen - allesamt Faktoren, die Korrelationen mit ehrenamtlichem Engagement erwarten lassen, gleichzeitig aber die Wahrscheinlichkeit, ehrenamtlich in einer Führungsposition aktiv zu sein, kaum beeinflussen sollten.

In die Hauptgleichung fließen zudem die engagementspezifischen Faktoren ein, die nur unter der Teilmenge der Engagierten erhoben wurden. Dazu zählen der Engagementbereich (Freizeit, Soziales, Politik, Glauben/Religion, Technik) und die Organisationsform (Verein, Partei/Verband, Kirche, Selbsthilfe, Ssonstige) (vgl. Tabelle 5 und 6 im Anhang für eine genauere Aufschlüsselung). Zudem wurden das Vorhandensein von Hauptamtlichen in der jeweiligen Organisation, die Häufigkeit der ehrenamtlichen Tätigkeit (täglich, wöchentlich, monatlich und seltener) sowie die Tätigkeitsdauer kontrolliert. Tabelle 1 gibt einen Überblick über die in der Hauptgleichung und der Auswahlgleichung verwendeten Variablen. 
Tabelle 1: Deskription der Modellvariablen

\begin{tabular}{|c|c|c|c|c|}
\hline & \multicolumn{2}{|c|}{ Hauptgleichung } & \multicolumn{2}{|c|}{ Auswahlgleichung } \\
\hline & Mittelwert & Std. Abw. & Mittelwert & Std. Abw. \\
\hline ehrenamtliches Engagement $(\mathrm{ja}=1)$ & - & - & 0,35 & 0,48 \\
\hline ehrenamtliche Leitungsposition $(\mathrm{ja}=1)$ & 0,33 & 0,47 & - & - \\
\hline Geschlecht $(F r a u=1)$ & 0,53 & 0,50 & 0,57 & 0,50 \\
\hline 14 bis 24 Jahre & 0,13 & 0,34 & 0,13 & 0,34 \\
\hline 25 bis 34 Jahre & 0,13 & 0,33 & 0,13 & 0,34 \\
\hline 35 bis 44 Jahre & 0,20 & 0,40 & 0,18 & 0,38 \\
\hline 45 bis 54 Jahre & 0,21 & 0,41 & 0,20 & 0,40 \\
\hline 55 bis 64 Jahre & 0,16 & 0,36 & 0,16 & 0,36 \\
\hline$>65$ Jahre & 0,17 & 0,38 & 0,20 & 0,40 \\
\hline Keine Kinder im Haushalt & 0,75 & 0,43 & 0,79 & 0,41 \\
\hline Kinder ohne Betreuungsaufwand & 0,05 & 0,21 & 0,04 & 0,19 \\
\hline Kinder mit tw. Betreuungsaufwand & 0,07 & 0,26 & 0,06 & 0,23 \\
\hline Kinder mit gänzl. Betreuungsaufwand & 0,13 & 0,34 & 0,11 & 0,31 \\
\hline kein Migrationshintergrund & 0,88 & 0,32 & 0,85 & 0,35 \\
\hline 1. Migrantengeneration & 0,03 & 0,17 & 0,05 & 0,21 \\
\hline 2. Migrantengeneration & 0,09 & 0,28 & 0,10 & 0,30 \\
\hline erwerbstätig & 0,55 & 0,50 & 0,51 & 0,50 \\
\hline arbeitslos & 0,04 & 0,19 & 0,05 & 0,22 \\
\hline nicht erwerbstätig & 0,05 & 0,22 & 0,05 & 0,22 \\
\hline Rentner & 0,21 & 0,41 & 0,25 & 0,43 \\
\hline sonstige Erwerbstätige & 0,15 & 0,36 & 0,14 & 0,35 \\
\hline noch Schüler & 0,07 & 0,25 & 0,07 & 0,25 \\
\hline Hauptschulabschluss & 0,12 & 0,32 & 0,19 & 0,39 \\
\hline Realschulabschluss & 0,28 & 0,45 & 0,30 & 0,46 \\
\hline Abitur & 0,53 & 0,50 & 0,44 & 0,50 \\
\hline Ostdeutschland & 0,23 & 0,42 & 0,26 & 0,44 \\
\hline Freizeitbereich & 0,38 & 0,49 & - & - \\
\hline sozialer Bereich & 0,30 & 0,46 & - & - \\
\hline politischer Bereich & 0,15 & 0,36 & - & - \\
\hline religiöser Bereich & 0,11 & 0,32 & - & - \\
\hline technischer Bereich & 0,05 & 0,22 & - & - \\
\hline Verein & 0,47 & 0,50 & - & - \\
\hline Partei/Verband & 0,11 & 0,31 & - & - \\
\hline Kirche & 0,12 & 0,33 & - & - \\
\hline Selbsthilfe/Projekt & 0,16 & 0,36 & - & - \\
\hline sonstige Einrichtungen & 0,14 & 0,35 & - & - \\
\hline täglich engagiert & 0,04 & 0,19 & - & - \\
\hline wöchentlich engagiert & 0,52 & 0,50 & - & - \\
\hline monatlich engagiert & 0,34 & 0,47 & - & - \\
\hline seltener/nie engagiert & 0,10 & 0,29 & - & - \\
\hline
\end{tabular}

Fortsetzung von Tabelle 1 auf der nächsten Seite 


\section{Fortsetzung Tabelle 1}

\begin{tabular}{|c|c|c|c|c|}
\hline & \multicolumn{2}{|c|}{ Hauptgleichung } & \multicolumn{2}{|c|}{ Auswahlgleichung } \\
\hline & Mittelwert & Std. Abw. & Mittelwert & Std. Abw. \\
\hline Hauptamtliche in Organisation $(\mathrm{ja}=1)$ & 0,15 & 0,36 & - & - \\
\hline aktiv $<3$ Jahre & 0,22 & 0,42 & - & - \\
\hline aktiv 3 bis 10 Jahre & 0,47 & 0,50 & - & - \\
\hline aktiv > 10 Jahre & 0,30 & 0,46 & - & - \\
\hline finanzielle Situation (sehr) gut & - & - & 0,41 & 0,49 \\
\hline finanzielle Situation befriedigend & - & - & 0,38 & 0,49 \\
\hline finanzielle Situation (sehr) schlecht & - & - & 0,20 & 0,40 \\
\hline Netzwerk klein & - & - & 0,32 & 0,47 \\
\hline Netzwerk mittel & - & - & 0,45 & 0,50 \\
\hline Netzwerk groß & - & - & 0,22 & 0,42 \\
\hline$<3$ Jahre am Ort & - & - & 0,09 & 0,28 \\
\hline 3 bis 10 Jahre am Ort & - & - & 0,20 & 0,40 \\
\hline$>10$ Jahre am Ort & - & - & 0,46 & 0,50 \\
\hline seit der Geburt am Ort & - & - & 0,25 & 0,43 \\
\hline nicht religiös & - & - & 0,45 & 0,50 \\
\hline schwach religiös & - & - & 0,18 & 0,39 \\
\hline mittel religiös & - & - & 0,24 & 0,43 \\
\hline stark religiös & - & - & 0,12 & 0,33 \\
\hline kein polit./gesell. Interesse & - & - & 0,14 & 0,35 \\
\hline polit./gesell. Interesse mittel & - & - & 0,46 & 0,50 \\
\hline polit./gesell. Interesse stark & - & - & 0,40 & 0,49 \\
\hline $\mathrm{N}$ & \multicolumn{2}{|c|}{6.996} & \multicolumn{2}{|c|}{19.995} \\
\hline
\end{tabular}

Quelle: Freiwilligensurvey 2009, eigene Berechnungen 


\section{Ergebnisse}

Tabelle 2 gibt einen Überblick über die Geschlechterverteilung im Datensatz sowie hinsichtlich des ehrenamtlichen Engagements im Allgemeinen und der Übernahme von ehrenamtlichen Leitungspositionen im Besonderen. Es zeigt sich, dass von den insgesamt 19.995 Personen 35 Prozent ( $N=6.996)$ ein Ehrenamt ausüben und davon wiederum 33 Prozent $(\mathrm{N}=2.286)$ eine ehrenamtliche Führungsposition bekleiden. Während 33 Prozent der Frauen sich ehrenamtlich engagieren, gehen 38 Prozent der Männer einer ehrenamtlichen Aktivität nach. Der Geschlechterunterschied fällt bei der Übernahme von Leitungspositionen in Ehrenämtern noch deutlicher aus. So übernehmen unter den ehrenamtlich aktiven Frauen nur 26 Prozent eine Führungsposition, während 40 Prozent der engagierten Männer ihr Ehrenamt in leitender Funktion ausüben.

Tabelle 2: Verteilung der Frauen und Männer auf Ehrenämter und Leitungspositionen in Ehrenämtern

\begin{tabular}{lcccccc}
\hline & \multicolumn{2}{c}{$\begin{array}{c}\text { Geschlechter- } \\
\text { verteilung }\end{array}$} & \multicolumn{2}{c}{$\begin{array}{c}\text { Ehrenamtliches } \\
\text { Engagement }\end{array}$} & \multicolumn{2}{c}{$\begin{array}{c}\text { Leitungspositionen } \\
\text { im Ehrenamt }\end{array}$} \\
& $\begin{array}{c}\text { In Pro- } \\
\text { zent }\end{array}$ & $\mathrm{N}$ & in Prozent & $\mathrm{N}$ & in Prozent & $\mathrm{N}$ \\
\hline Frauen & 57 & 11.341 & 33 & 3.717 & 26 & 969 \\
Männer & 43 & 8.654 & 38 & 3.279 & 40 & 1.317 \\
\hline Gesamt & 100 & 19.995 & 35 & 6.996 & 33 & 2.286 \\
\hline
\end{tabular}

Quelle: Freiwilligensurvey 2009, eigene Berechnungen

Das Ziel der Analyse ist, mittels Probit-Regressionen zu bestimmen, welche Faktoren eine Übernahme der Führungspositionen in Ehrenämtern vorhersagen können und inwiefern eine Geschlechterungleichheit auch unter Kontrolle weiterer Merkmale bestehen bleibt. Es wurden für die Gesamtpopulation, für Männer und für Frauen jeweils zwei Modelle einmal mit und einmal ohne Selektion geschätzt. Die Ergebnisse dieser ProbitRegressionen sind in Tabelle 3 dokumentiert. Bei den Modellen mit Selektion werden ausschließlich die Ergebnisse der Variablen in der Hauptgleichung sowie die entsprechenden Teststatistiken berichtet. Die Ergebnisse der vorgeschalteten Auswahlgleichung sind in Tabelle 4 im Anhang dokumentiert. 
Tabelle 3: Leitungspositionen in Ehrenämtern, average marginal effects der Probit Regressionen mit und ohne Heckman-Selektion

\begin{tabular}{|c|c|c|c|c|c|c|}
\hline & \multirow{2}{*}{\multicolumn{2}{|c|}{ Gesamtmodell }} & \multicolumn{2}{|c|}{ Frauen } & \multicolumn{2}{|c|}{ Männer } \\
\hline & & & \multicolumn{2}{|c|}{ Selektion } & \multicolumn{2}{|c|}{ Selektion } \\
\hline & ohne & mit & ohne & mit & ohne & mit \\
\hline \multicolumn{7}{|l|}{ Geschlecht } \\
\hline Frau (Ref.: Mann) & $-0,091^{* * *}$ & $-0,079^{* * * *}$ & & & & \\
\hline \multicolumn{7}{|l|}{ Alter } \\
\hline 14 bis 24 Jahre & $-0,037$ & $-0,019$ & 0,024 & 0,044 & $-0,100^{*}$ & $-0,072$ \\
\hline 25 bis 34 Jahre & $-0,071^{* * *}$ & $-0,045^{*}$ & $-0,034$ & $-0,002$ & $-0,110^{* * *}$ & $-0,082^{* *}$ \\
\hline 35 bis 44 Jahre & $-0,056^{* *}$ & $-0,054^{* *}$ & $-0,040$ & $-0,040$ & $-0,073^{* *}$ & $-0,062^{*}$ \\
\hline 45 bis 54 Jahre & Ref. & Ref. & Ref. & Ref. & Ref. & Ref. \\
\hline 55 bis 64 Jahre & 0,022 & 0,022 & 0,009 & 0,010 & 0,040 & 0,033 \\
\hline $65+$ Jahre & 0,041 & 0,053 & 0,004 & 0,013 & $0,092^{*}$ & $0,100^{*}$ \\
\hline \multicolumn{7}{|l|}{ Elternschaft } \\
\hline keine Kinder & Ref. & Ref. & Ref. & Ref. & Ref. & Ref. \\
\hline $\begin{array}{l}\text { Kinder ohne Betreu- } \\
\text { ungsaufwand }\end{array}$ & 0,023 & 0,001 & 0,055 & 0,058 & 0,021 & $-0,013$ \\
\hline $\begin{array}{l}\text { Kinder mit tw. Betreu- } \\
\text { ungsaufwand }\end{array}$ & $-0,027$ & $-0,059^{*}$ & $-0,045$ & $-0,088^{*}$ & $-0,016$ & $-0,047$ \\
\hline $\begin{array}{l}\text { Kinder mit gänzl. Be- } \\
\text { treuungsaufwand }\end{array}$ & $-0,008$ & $-0,043^{*}$ & $-0,022$ & $-0,062^{*}$ & 0,009 & $-0,018$ \\
\hline \multicolumn{7}{|l|}{ Migrationshint. } \\
\hline kein Migrationshint. & Ref. & Ref. & Ref. & Ref. & Ref. & Ref. \\
\hline 1. Mig.generation & $-0,063$ & $-0,007$ & $-0,031$ & 0,024 & $-0,102$ & $-0,028$ \\
\hline 2. Mig.generation & 0,006 & 0,027 & 0,046 & $0,071^{*}$ & $-0,043$ & $-0,014$ \\
\hline \multicolumn{7}{|l|}{ Erwerbsstatus } \\
\hline erwerbstätig & Ref. & Ref. & Ref. & Ref. & Ref. & Ref. \\
\hline arbeitslos & $-0,065^{*}$ & $-0,043$ & $-0,056$ & $-0,039$ & $-0,076$ & $-0,046$ \\
\hline nicht erwerbstätig & $-0,010$ & $-0,012$ & 0,011 & 0,010 & $-0,166$ & $-0,181$ \\
\hline Rentner/Pensionär & $-0,053^{*}$ & $-0,048$ & $-0,029$ & $-0,025$ & $-0,087^{*}$ & $-0,077^{*}$ \\
\hline Sonst. erwerbstätig & $-0,054^{*}$ & $-0,065^{*}$ & $-0,091^{* *}$ & $-0,111^{* *}$ & $-0,016$ & $-0,027$ \\
\hline noch Schüler & $0,067^{*}$ & $0,071^{*}$ & 0,069 & 0,070 & 0,052 & 0,061 \\
\hline \multicolumn{7}{|l|}{ Qualifikation } \\
\hline Hauptschule & $-0,033$ & $-0,000$ & $-0,015$ & 0,024 & $-0,064^{*}$ & $-0,029$ \\
\hline Realschule & Ref. & Ref. & Ref. & Ref. & Ref. & Ref. \\
\hline Abitur & $0,049^{* * *}$ & 0,024 & $0,057^{* * * *}$ & 0,032 & 0,029 & 0,004 \\
\hline
\end{tabular}

Forstetzung Tabelle 3 auf der nächsten Seite 
Fortsetzung von Tabelle 3

\begin{tabular}{|c|c|c|c|c|c|c|}
\hline & \multicolumn{2}{|c|}{ Gesamtmodell } & \multicolumn{2}{|c|}{ Frauen } & \multicolumn{2}{|c|}{ Männer } \\
\hline & \multicolumn{2}{|c|}{ Selektion } & \multicolumn{2}{|c|}{ Selektion } & \multicolumn{2}{|c|}{ Selektion } \\
\hline & ohne & mit & ohne & mit & ohne & mit \\
\hline \multicolumn{7}{|l|}{ Hauptamtl. in Org. } \\
\hline ja (Ref.: nein) & $-0,131^{* * *}$ & $-0,135^{* * *}$ & $-0,108^{* * *}$ & $-0,121^{* * *}$ & $-0,164^{* * *}$ & $-0,151^{* * *}$ \\
\hline \multicolumn{7}{|l|}{ Aktivitätshäufigkeit } \\
\hline täglich aktiv & $0,127^{* * *}$ & $0,128^{* * * *}$ & $0,124^{* * *}$ & $0,139^{* *}$ & $0,127^{* *}$ & $0,111^{* *}$ \\
\hline wöchentlich aktiv & Ref. & Ref. & Ref. & Ref. & Ref. & Ref. \\
\hline monatlich aktiv & $-0,070^{* * * *}$ & $-0,069^{* * * *}$ & $-0,020$ & $-0,019$ & $-0,129^{* * * *}$ & $-0,116^{* * *}$ \\
\hline seltener aktiv & $-0,299^{* * *}$ & $-0,301^{* * *}$ & $-0,223^{* * *}$ & $-0,244^{* * *}$ & $-0,403^{* * *}$ & $-0,362^{* * *}$ \\
\hline \multicolumn{7}{|l|}{ Engagementdauer } \\
\hline aktiv $<3$ Jahre & $-0,059^{* * *}$ & $-0,057^{* * * *}$ & $-0,072^{* * * *}$ & $-0,076^{* * *}$ & $-0,041$ & $-0,036$ \\
\hline aktiv seit 3-10 Jahren & Ref. & Ref. & Ref. & Ref. & Ref. & Ref. \\
\hline aktiv $>$ 10 Jahre & 0,017 & 0,013 & 0,002 & $-0,003$ & 0,026 & 0,022 \\
\hline \multicolumn{7}{|l|}{ Region } \\
\hline Ostdeut. (Ref.: West) & $-0,023$ & $-0,003$ & $-0,004$ & 0,016 & $-0,048^{*}$ & $-0,021$ \\
\hline \multicolumn{7}{|l|}{ Engagementbereich } \\
\hline Freizeitbereich & Ref. & Ref. & Ref. & Ref. & Ref. & Ref. \\
\hline sozialer Bereich & 0,008 & 0,011 & 0,008 & 0,015 & 0,009 & 0,012 \\
\hline politischer Bereich & 0,008 & 0,007 & 0,024 & 0,029 & $-0,012$ & $-0,017$ \\
\hline religiöser Bereich & 0,028 & 0,007 & 0,023 & 0,003 & 0,039 & 0,016 \\
\hline technischer Bereich & 0,028 & 0,028 & 0,000 & $-0,000$ & 0,026 & 0,022 \\
\hline \multicolumn{7}{|l|}{ Organisationsform } \\
\hline Verein & Ref. & Ref. & Ref. & Ref. & Ref. & Ref. \\
\hline Partei & $0,107^{* * * *}$ & $0,106^{* * *}$ & $0,078^{* *}$ & $0,083^{* *}$ & $0,149^{* * * *}$ & $0,138^{* * *}$ \\
\hline Kirche & $-0,025$ & $-0,046$ & $-0,034$ & $-0,059$ & $-0,007$ & $-0,026$ \\
\hline Selbsthilfegruppe & $-0,107^{* * *}$ & $-0,107^{* * * *}$ & $-0,110^{* * * *}$ & $-0,123^{* * * *}$ & $-0,097^{* * *}$ & $-0,083^{* *}$ \\
\hline sonstige & $-0,064^{* * *}$ & $-0,066^{* * *}$ & $-0,088^{* * *}$ & $-0,100^{* * *}$ & $-0,028$ & $-0,026$ \\
\hline$N$ & 6.996 & 6.996 & 3.717 & 3.717 & 3.278 & 3.279 \\
\hline$N$ (zensiert) & & 12.999 & & 7.624 & & $5 \cdot 375$ \\
\hline rho & & $-0,46^{* * *}$ & & $-0,45^{* * *}$ & & $-0,51^{* * *}$ \\
\hline Wald-Chi² & & $\begin{array}{c}575,09 \\
(40)\end{array}$ & & $\begin{array}{c}268,98 \\
(39)\end{array}$ & & $\begin{array}{c}263,81 \\
(39)\end{array}$ \\
\hline pseudo $R^{2}$ & 0,092 & & 0,073 & & 0,091 & \\
\hline
\end{tabular}


Bevor wir auf die uns eigentlich interessierenden Geschlechterunterschiede eingehen, zeigt ein Blick auf die Ergebnisse des Gesamtmodells (Tabelle 3), welche Determinanten unabhängig vom Geschlecht mit der Bekleidung ehrenamtlicher Führungspositionen in Zusammenhang stehen. So ist ein u-förmiger Alterseffekt zu beobachten, wobei die für Männer und Frauen getrennt vorgenommenen Schätzungen zeigen, dass dies insbesondere ein für Männer zu beobachtendes Phänomen ist. Zudem zeigt sich ein klarer positiver Bildungszusammenhang nur in der Schätzung ohne Selektion während bei der Schätzung unter Berücksichtigung der Heckman-Korrektur dieser Bildungsgradient verschwindet. Dass wir eine größere Zahl besser Qualifizierte unter Vereinsvorsitzenden usw. finden, ist also auf ein generell mit zunehmender Bildung wachsendes ehrenamtliches Engagement zurückzuführen. Sobald sich geringer Qualifizierte unentgeltlich engagieren, haben diese im Schnitt ähnlich große Chancen, ehrenamtliche Leitungsaufgaben zu übernehmen wie ihre besser qualifizierten Vereinskollegen oder Parteigenossen. Gleichzeitig ist kein (klarer) Zusammenhang zwischen ehrenamtlichen Leitungsaktivitäten und dem Erwerbs-, bzw. Migrationsstatus nachweisbar. Werden jedoch schulpflichtige Kinder betreut, senkt dies die Wahrscheinlichkeit signifikant, eine ehrenamtliche Leitungsaufgabe zu übernehmen. Dieser im Gesamtmodell zu beobachtende Zusammenhang ist jedoch in erster Linie auf Frauen zurückzuführen, denn die getrennten Schätzungen zeigen, dass zwischen Vätern und kinderlosen Männern keine Unterschiede bei der Bekleidung von ehrenamtlichen Führungsfunktionen existieren.

Darüber hinaus gibt es eine deutliche positive Korrelation zwischen der Engagementhäufigkeit und der Bekleidung von Führungspositionen. Weiterhin ist zu sehen, dass eine erst relativ kurze Engagementkarriere ( $<3$ Jahre) signifikant seltener bereits zu Leitungspositionen geführt hat. Neben diesen individuellen sozioökonomischen bzw. soziodemographischen Determinanten sowie Charakteristika des individuellen Engagementverhaltens lassen sich auch Zusammenhänge zwischen den Merkmalen der Organisation und der Wahrscheinlichkeit, dass Menschen dort ehrenamtliche Führungspositionen bekleiden, feststellen. Sind neben Ehrenamtlichen auch Hauptamtliche beschäftigt, verringert dies die Wahrscheinlichkeit, dass ein unter diesen Bedingungen aktiver Ehrenamtlicher eine Leitungsaufgabe übernimmt. Zudem zeigt sich, dass offenbar gerade Parteien und Verbände im Vergleich zu Vereinen signifikant häufiger ehrenamtliche Führungskräfte haben. Hingegen ist für unentgeltlich in der Selbsthilfe und sonstigen Organisationen Tätige im Vergleich zu Vereinsaktiven unwahrscheinlicher, dass sie eine Führungsfunktion innehaben.

Betrachtet man nun den für unsere Fragestellung zentralen Aspekt des Zusammenhangs zwischen Geschlecht und ehrenamtlichen Leitungsfunktionen, so zeigt sich anhand der signifikant negativen marginalen Effekte, dass selbst unter Kontrolle vorgelagerter Selektionseffekte und wesentlicher individueller und organisationaler Einflussfaktoren Frauen deutlich seltener ehrenamtliche Führungspositionen bekleiden als Männer. Wir finden also selbst in dem Bereich, in dem Frauen aufgrund ihrer Ausbildung und ihrer 
tatsächlich oder vermeintlich besseren sozialen Kompetenzen gegenüber Männern bevorteilt sein sollten, eine ähnliche Gleichstellungsproblematik wie im Erwerbsleben. Unabhängig von diesem generellen Unterschied unterscheiden sich Männer und Frauen dann jedoch kaum hinsichtlich der Faktoren, die die Übernahme einer unbezahlten Leitungstätigkeit unterstützen bzw. behindern.

Abbildung 1: Average marginal effects für Frauen, eine Leitungsposition im genannten Engagementbereich oder der jeweiligen Organisationsform zu bekleiden

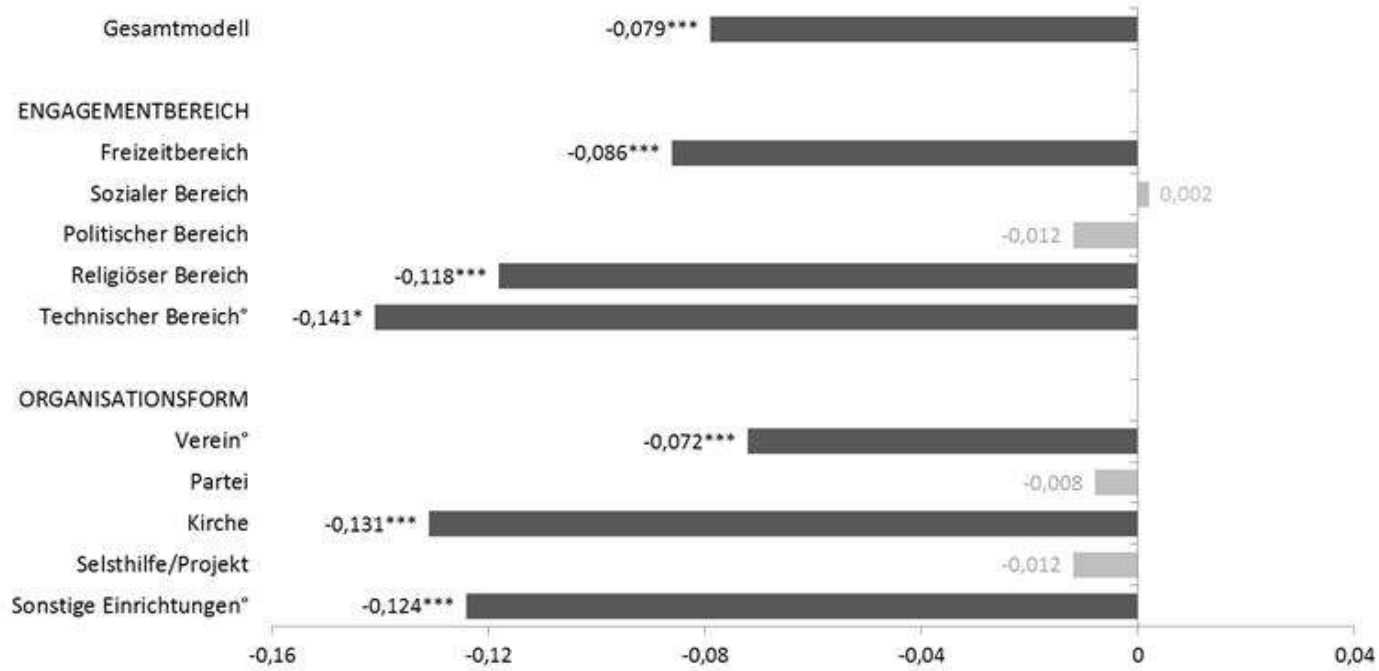

Quelle: Freiwilligensurvey 2009, eigene Berechnungen

Kontrollvariablen (Hauptgleichung): Alter, zu betreuende Kinder im Haushalt, Migrationshintergrund, Erwerbsstatus, höchster Schulabschluss, Engagementhäufigkeit, Tätigkeitsdauer, Vorhandensein der Hauptamtlichen und Region; Kontrollvariablen (Auswahlgleichung): Geschlecht, Alter, Kinder im Haushalt, Migrationshintergrund, Erwerbsstatus, höchster Schulabschluss, Religiosität, Interesse an politischen und gesellschaftlichen Themen, Region.

${ }^{*} \mathrm{p}<0.05,{ }^{* *} \mathrm{p}<0.01,{ }^{* * *} \mathrm{p}<0.001$; hellen Balken = nicht sig. Effekte

${ }^{\circ}$ kein Sample Selektion Bias; Ergebnisse der Probit Regressionen ohne Selektion.

Um zu überprüfen, inwieweit ein Zusammenhang mit dem Engagementbereich bzw. der Organisationsform und der Benachteiligung von Frauen bei der Besetzung ehrenamtlicher Leitungsfunktionen auch unter Berücksichtigung wesentlicher Kontrollvariablen existiert, haben wir erneut unterschiedliche Selektionsmodelle für die entsprechenden Bereiche und Organisationsformen geschätzt. Die Frage, die damit beantwortet werden soll, lautet, ob die Unterrepräsentanz von Frauen in Führungspositionen unter anderem auch ein Ergebnis einer geschlechtsspezifischen Entscheidung für bestimmte Inhalte oder Organisationsformen ist. Alternativ ist auch denkbar, dass diese Selektivität nicht oder nur zum Teil die Benachteiligung von Frauen erklären kann und es somit zu einer direkten Diskriminierung von Frauen zumindest in einzelnen Engagementbereichen und Organisationsformen kommt. Abbildung 1 dokumentiert die entsprechenden Ergeb- 
nisse der Heckprob-Schätzungen. Aus Gründen der Übersichtlichkeit werden hier lediglich die durchschnittlichen marginalen Effekte für Frauen im Vergleich zu Männern gezeigt, eine Führungsposition im jeweiligen Bereich zu übernehmen.

Wir sehen in Abbildung 1, dass sich nur in einigen Bereichen bzw. bestimmten Organisationsformen eine Benachteiligung von Frauen bei der Besetzung von Führungspositionen nachweisen lässt. Dies ist im Freizeitbereich und im religiösen und technischen Bereich der Fall und zeigt sich insbesondere in Vereinen, Kirchen und sonstigen Einrichtungen. Bei Parteien und in Selbsthilfegruppen lassen sich nach Kontrolle individuellen Unterschiede und nach Berücksichtigung möglicher Selektionseffekte hingegen keine Geschlechterunterschiede bei der Besetzung von Leitungsfunktionen feststellen. Gleiches gilt für den politischen und technischen Engagementbereich. Die Befunde machen deutlich, dass Tätigkeitsinhalte sowie die Organisationsform zu einer mehr oder weniger starken Benachteiligung von Frauen bei der Besetzung von ehrenamtlichen Führungsaufgaben beitragen können.

\section{Zusammenfassung und Diskussion}

Frauen weisen im Vergleich zu Männern eine deutlich geringere Wahrscheinlichkeit auf, in einer ehrenamtlichen Tätigkeit Führungspositionen zu übernehmen. Dieser Zusammenhang ist auch dann stabil, wenn (Selbst-)Selektionseffekte sowie eine Reihe zentraler soziodemografischer, sozioökonomischer und engagementspezifischer Faktoren berücksichtigt werden. Eine differenzierte Analyse zeigt zudem, dass eine Benachteiligung von Frauen hinsichtlich der Übernahme ehrenamtlicher Leitungsfunktionen insbesondere für bestimmte Tätigkeitsbereiche bzw. in bestimmten Organisationsformen nachweisbar ist. Vor allem Vereine und Kirchen bzw. religiöse Organisationen zeigen hier deutliche Defizite bei der Gleichstellung. Immerhin sind hier insgesamt knapp 6o Prozent aller Ehrenamtlichen in Deutschland aktiv. Daher können Vereine und Kirchen aufgrund ihrer quantitativen Bedeutung zusammen mit der heterogenen Gruppe der sonstigen Organisationen als Treiber der insgesamt zu beobachtenden Benachteiligung von Frauen als ehrenamtliche Führungskräfte identifiziert werden.

Die Ursachen dafür, dass Frauen seltener ehrenamtliche Führungsaufgaben übernehmen, dürften allerdings keineswegs allein in der Form der jeweiligen gemeinnützigen Organisation oder aber der inhaltlichen Tätigkeiten liegen (vgl. zu einer ähnlichen Befundlage in den USA Rotolo \& Wilson, 2007). Denkbar wäre nämlich darüber hinaus, dass Führungspositionen in Ehrenämtern von jenen Personen übernommen werden, die in ihrem Erwerbsleben solche Positionen schon bekleiden (Spill-over-Effekt). Hier scheinen Männer durch ihre Überrepräsentanz in den Führungsetagen in Unternehmen bevorteilt zu sein, weil sie ihre schon geübte und vertraute Rolle aus dem Erwerbsleben in den Bereich des Ehrenamts direkt mitnehmen können. Nicht zuletzt weist die verringerte Wahrscheinlichkeit, beim Einstieg in die ehrenamtliche Tätigkeit sofort eine Führungsaufgabe zu übernehmen, welche nur Frauen betrifft und für Männer keine Rolle 
spielt, auf mögliche strukturelle und ideologische Barrieren hin. Frauen müssen sich insofern auch im Ehrenamt erst einmal „beweisen“ oder „bewähren“, während Männern eine Führungsaufgabe sofort zugetraut wird. Insgesamt scheint der Bereich unbezahlten sozialen Engagements für Frauen jedenfalls kein alternatives Betätigungsfeld zu bieten, in dem sie die Defizite bei der Bekleidung von Leitungsfunktionen im Erwerbsleben kompensieren könnten.

Jedoch ist als weitere Begründung durchaus denkbar, dass die seltenere Übernahme von ehrenamtlichen Leitungsfunktionen durch Frauen nicht (allein) auf strukturelle Diskriminierung „von außen“ zurückzuführen ist, sondern zu einem mehr oder weniger großen Teil auch durch einen freiwilligen Verzicht von Frauen auf Führungspositionen erklärt werden könnte; hierbei wäre dann auch die Problematik der Vereinbarkeit von Familie, Beruf und leitendem Ehrenamt zu berücksichtigen. Denn in dem Maß, in dem nach wie vor insbesondere Frauen die Verantwortung für Familie und Haushalt zugesprochen wird, könnte dies bei den Frauen im Zweifel eher zu einem Verzicht auf tendenziell zeitintensive Führungsaufgaben gerade im Bereich unbezahlten ehrenamtlichen Engagements führen. Der Befund, dass erstens Mütter seltener ehrenamtliche Leitungsfunktionen übernehmen als Frauen ohne Betreuungsaufgabe und sich zweitens bei Männern kein solcher Zusammenhang nachweisen lässt, deutet in diese Richtung.

Allerdings zeigt sich darüber hinaus weder für Männer noch für Frauen ein Unterschied zwischen erwerbstätigen und nicht-erwerbstätigen Aktiven. Unbezahlte Führungsfunktionen zu übernehmen ist also nicht alleine eine Frage frei verfügbarer Zeit. Einschränkend muss man aber hierbei auch darauf hinweisen, dass sich diese Aussagen auf relativ wenige und dazu noch grobe Indikatoren innerhalb des Freiwilligensurveys stützen. Vor dem Hintergrund, dass davon ausgegangen wird, dass Führungsaufgaben mit erhöhtem zeitlichem Aufwand einhergehen, wäre es wünschenswert gewesen, neben Indikatoren der Haushaltszusammensetzung auch den Umfang der Erwerbstätigkeit zu berücksichtigen. Des Weiteren würde ein Indikator zur Bekleidung von Führungsposition im Erwerbsleben die Aussagekraft der Modelle erheblich verbessern (vgl. dazu auch Marshall \& Taniguchi, 2012). Hier könnten wir ausgehend von indirekten Merkmalen lediglich Parallelen zwischen der Positionierung im Erwerbsleben und im Ehrenamt vermuten. Leider sind beide erwähnten Indikatoren im Freiwilligensurvey bislang nicht enthalten. Es bleibt abzuwarten, ob die gewünschten Analysen mit der aktuellen Welle des Freiwilligensurveys 2014 zu realisieren sind.

Neben den neuen Erkenntnissen hinsichtlich der Diskriminierung von Frauen im Bereich des unbezahlten ehrenamtlichen Engagements, lassen sich unserer Ansicht nach zudem interessante generelle Rückschlüsse für die derzeit in Deutschland intensiv geführte Debatte um Frauen in Führungspositionen ziehen. Wie zu Beginn ausgeführt wurde, ist ein populäres Argument für das Fehlen von weiblichen Führungskräften in Wirtschaftsunternehmen deren angeblich inadäquate Ausbildung und mangelnde berufliche Passung. Wenn dies tatsächlich der wesentliche Grund für den Männerüberschuss in den Führungsetagen wäre, so müsste dieser Nachteil im Bereich des nicht gewinnorientierten Non-Profit-Sektors zumindest ausgeglichen werden. Dies ist aber offensichtlich nur sehr eingeschränkt der Fall. Zumindest bei Leitungsfunktionen in Kirchen und Vereinen sehen wir hinsichtlich der Benachteiligung von Frauen ein ähnliches Bild wie 
in For-Profit-Unternehmen. Dies sind wichtige Hinweise darauf, dass strukturelle und organisatorische Faktoren eine wesentliche Bedeutung für die Erklärung und mithin auch für eine zukünftige Beseitigung der auch im Bereich des Ehrenamtes vielfach existierenden Gläsernen Decke zukommt. Zudem bleibt abzuwarten, inwieweit ein fortschreitender Abbau der Diskriminierung von Frauen im Erwerbsleben sowie damit einhergehende, sich langsam wandelnde Rollenbilder durch entsprechende Spill-OverEffekte auch zu einem Abbau der geschlechtsspezifischen Unterschiede bei der Besetzung unbezahlter ehrenamtlicher Führungspositionen in Zukunft beitragen wird. 


\section{Literaturverzeichnis}

Achatz, J. (2008a). Die Integration von Frauen in Arbeitsmärkten und Organisationen. In S. M. Wilz (Hg.), Geschlechterdifferenzen - Geschlechterdifferenzierungen. Ein Überblick über gesellschaftliche Entwicklungen und theoretische Positionen (S. 105138). Wiesbaden: VS Verl. für Sozialwiss.

Achatz, J. (2008b). Geschlechtersegregation am Arbeitsmarkt. In M. Abraham \& T. Hinz (Hg.), Arbeitsmarktsoziologie. Probleme, Theorien, empirische Befunde (pp. 263-301). Wiesbaden: VS. Verlag für Sozialwissenschaften.

Alemann, A. von. (2007). Chancenungleichheit im Management: Begründungsmuster der Unterrepräsentanz von Frauen in Führungspositionen der Wirtschaft. Sozialwissenschaften und Berufspraxis, 3o(1), 21-38.

Arminger, G. (1996). The Analysis of Panel Data with Nonmetric Variables: Probit Models and a Heckman Correction for Selectivity Bias. In U. Engel \& J. Reinecke (Hg.), Analysis of Change: Advanced Techniques in Panel Data Research (S. 61-85). Berlin: Walter de Gruyter \& Co.

Bauernschuster, S., \& Fichtl, A. (2013). Brauchen wir eine gesetzliche Frauenquote? ifo Schnelldienst, 66(2), 39-48.

BDA \& BDI - Abteilung Arbeits- und Tarifrecht \& Abteilung Recht und Versicherung. (2015). Geschlechterquote: Richtiges Ziel, falscher Weg: Stellungnahme zum Regierungsentwurf eines "Gesetzes zur gleichberechtigten Teilhabe von Frauen und Männern an Führungspositionen in der Privatwirtschaft und im öffentlichen Dienst".

Bechmann, S., Dahms, V., Tschersich, N., Frei, M., Leber, U., \& Schwengler, B. (2013). Beschäftigungsmuster von Frauen und Männern. Auswertungen des IAB-Betriebspanels 2012. In Institut für Arbeitsmarkt- und Berufsforschung der Bundesagentur für Arbeit (Hg.), IAB Forschungsbericht. Nürnberg.

Beck, U. (1999). Schöne neue Arbeitswelt: Vision: Weltbürgergesellschaft. Visionen für das 21. Jahrhundert: 2. Aufl. Frankfurt/Main: Campus.

Bundesministerium für Familie, Senioren, Frauen und Jugend (BMFSFJ). (2010). Hauptbericht des Freiwilligensurveys 2009: Ergebnisse der repräsentativen Trenderhebung zu Ehrenamt, Freiwilligenarbeit und Bürgerschaftlichem Engagement. Berlin.

Busch, A. (2013). Der Einfluss der beruflichen Geschlechtersegregation auf den "Gender Pay Gap": Zur Bedeutung geschlechtlich konnotierter Arbeitsinhalte. Kölner Zeitschrift für Soziologie und Sozialpsychologie, 65(2), 301-338.

Busch, A., \& Holst, E. (2008). Verdienstdifferenzen zwischen Frauen und Männern nur teilweise durch Strukturmerkmale zu erklären. DIW Wochenbericht, (15), 184-190.

Daniels, A. K. (1988). Invisible Careers: Women Civic Leaders from the Volunteer World. Women in Culture and Society. Chicago: University of Chicago Press.

Erlinghagen, M. (2000). Informelle Arbeit: Ein Überblick über einen schillernden Begriff. Schmollers Jahrbuch. Zeitschrift für Wirtschafts- und Sozialwissenschaften, $120(2), 239-274$. 
Erlinghagen, M. (2003). Die individuellen Erträge ehrenamtlicher Arbeit: Zur sozioökonomischen Theorie unentgeltlicher, haushaltsextern organisierter Produktion. Kölner Zeitschrift für Soziologie und Sozialpsychologie, 55(4), 737-757.

Erlinghagen, M. (2013). Ehrenamt. In S. Mau \& N. M. V. Schöneck (Hg.), Handwörterbuch zur Gesellschaft Deutschlands (S. 199-212). Wiesbaden: VS Verl. für Sozialwiss.

Gensicke, T. (2011). Freiwilligensurvey. In T. Olk \& B. Hartnuß (Hg.), Handbuch bürgerschaftliches Engagement (S. 691-704). Weinheim: Beltz Juventa.

Heckman, J. J. (1979). Sample Selection Bias as a Specification Error. Econometrica, 47(1), 153-161.

Helms, S., \& McKenzie, T. (2014). Gender Differences in Formal and Informal Volunteering in Germany. VOLUNTAS: International Journal of Voluntary and Nonprofit Organizations, 25(4), 887-904.

Hinz, T., \& Auspurg, K. (2010). Geschlechtsbezogene Diskriminierung bei der Entlohnung. In U. Hormel \& A. Scherr (Hg.), Diskriminierung. Grundlagen und Forschungsergebnisse (S. 135-149). Wiesbaden: VS Verl. für Sozialwiss.

Holst, E. (2008). Führungskräfte-Monitor 2001 - 2006. Forschungsreihe des Bundesministeriums für Familie, Senioren, Frauen und Jugend: Band 7. Baden-Baden: Nomos Verlag.

Holst, E., \& Kirsch, A. (2014). Frauen sind in Vorständen großer Unternehmen in Deutschland noch immer die Ausnahme: moderat steigende Anteile in Aufsichtsräten. DIW Wochenbericht, (3), 19-31.

Holst, E., Busch, A., \& Kröger, L. (2012). Führungskräfte-Monitor 2012: Update 2001 2010. Berlin: DIW Berlin.

Jann, B. (2008). Lohngerechtigkeit und Geschlechterdiskriminierung. Evidenz aus einem Vignetten-Experiment. In B. Jann (Hg.), Erwerbsarbeit, Einkommen und Geschlecht. Studien zum Schweizer Arbeitsmarkt (S. 107-126). Wiesbaden: VS Verl. für Sozialwiss.

Kleinert, C. (2006). Frauen in Führungspositionen: Karriere mit Hindernissen. Das familiäre Umfeld und die traditionelle Arbeitsteilung sind für den beruflichen Aufstieg von Frauen nach wie vor ein größeres Risiko als für den von Männern (IAB Kurzbericht No. 9). Nürnberg.

Kleinert, C., Kohaut, S., Brader, D., \& Lewerenz, J. (2007). Frauen an der Spitze - Arbeitsbedingungen und Lebenslagen weiblicher Führungskräfte. Frankfurt am Main u.a.: Campus-Verl.

Klenner, C., Pfahl, S., \& Seifert, H. (2001). Ehrenamt und Erwerbsarbeit - Zeitbalance oder Zeitkonkurrenz?: Forschungsprojekt im Auftrag des Ministeriums für Arbeit und Soziales, Qualifikation und Technologie des Landes Nordrhein-Westfalen. Düsseldorf: SCHÄFER GRAPHICS Agentur und Verlag GmbH.

Kohaut, S., \& Möller, I. (2013). Frauen in Führungspositionen: Punktgewinn in westdeutschen Großbetrieben (No. 23). Nürnberg.

Krell, G. (2010). Führungspositionen. In Projektgruppe GiB (Hg.), Forschung aus der Hans-Böckler-Stiftung: Vol. 110. Geschlechterungleichheiten im Betrieb. Arbeit, Entlohnung und Gleichstellung in der Privatwirtschaft (S. 423-484). Berlin: edition sigma. 
Maier, F., Stettes, O., \& Queisser, M. (2013). Geschlechterunterschiede auf dem Arbeitsmarkt sind eine zentrale Ursache der Lohnlücke. ifo Schnelldienst, 66(7), 3-6.

Marshall, G. A., \& Taniguchi, H. (2012). Good Jobs, Good Deeds: The Gender-Specific Influences of Job Characteristics on Volunteering. VOLUNTAS: International Journal of Voluntary and Nonprofit Organizations, 23(1), 213-235.

Müller, U. (1999). Zwischen Licht und Grauzone. Frauen in Führungspositionen. Arbeit. Zeitschrift für Arbeitsforschung, Arbeitsgestaltung und Arbeitspolitik, 8(2), 137-161.

Muro, J., Suárez, C., \& Zamora, María del Mar. (2010). Computing Murphy-Topel-corrected variances in a heckprobit model with endogeneity. The Stata Journal, 1O(2), 252258.

Notz, G. (2006). EhrenAMT und Arbeit - zwischen Verantwortung und Verpflichtung. In S. Mühlpfordt \& P. Richter (Hg.), Ehrenamt und Erwerbsarbeit (S. 70-81). München und Mering: Rainer Hampp Verlag.

OECD. (2013). Jobs and wages (OECD Factbook 2013: Economic, Environmental and Social Statistics). Paris.

Ohlendieck, L. (2003). Die Anatomie des Glashauses: Ein Beitrag zum Verständnis des Glass-Ceiling-Phänomens. In U. Pasero (Hg.), Gender - from Costs to Benefits (S. 183193). Wiesbaden: VS Verlag für Sozialwissenschaften.

Ott, N. (1993). Die Rationalität innerfamilialer Entscheidungen als Beitrag zur Diskriminierung weiblicher Arbeit. In G. Grözinger, R. Schubert, \& J. G. Backhaus (Hg.), Jenseits von Diskriminierung. Zu den institutionellen Bedingungen weiblicher Arbeit in Beruf und Familie (S. 113-146). Marburg: Metropolis.

Penner, A. M. (2008). Gender Differences in Extreme Mathematical Achievement: An International Perspective on Biological and Social Factors. American Journal of Sociology, 114, S138-S170.

Picot, S., \& Gensicke, T. (2005). Freiwilliges Engagement bei Frauen und Männern im Zeitvergleich 1999 - 2004. In Bundesministerium für Familie, Senioren, Frauen und Jugend (Hg.), Freiwilliges Engagement in Deutschland 1999-2004. Ergebnisse der repräsentativen Trenderhebung zu Ehrenamt, Freiwilligenarbeit und bürgerschaftlichem Engagement (S. 258-302). München,

Promberger, M. (2008). Arbeit, Arbeitslosigkeit und soziale Integration. Aus Politik und Zeitgeschichte, 40-41, 7-15.

Puhani, P. (2000). The Heckman Correction for Sample Selection and Its Critique. Journal of Economic Surveys, 14(1), 53-68.

Reskin, B. F. (1994). Sex Segregation: Explaining Stability and Change in the Sex Composition of Work. In P. Beckmann \& G. Engelbrech (Hg.), Beiträge zur Arbeitsmarktund Berufsforschung: Nr. 179. Arbeitsmarkt für Frauen 2000 - ein Schritt vor oder ein Schritt zurück? Kompendium zur Erwerbstätigkeit von Frauen (S. 97-115). Nürnberg: IAB.

Rotolo, T., \& Wilson, J. (2007). Sex Segregation in Volunteer Work. The Sociological Quarterly, 48(3), 559-585.

Sing, D., \& Hilpert, M. (2002). Frauen zwischen Erwerbstätigkeit, Ehrenamt und Familienarbeit. In E. Kistler, H.-H. Noll, \& E. Priller (Hg.), Perspektiven gesellschaftlichen 
Zusammenhalts. Empirische Befunde, Praxiserfahrungen, Messkonzepte (S. 325-341). Berlin: edition sigma.

Son, J., \& Wilson, J. (2010). Genetic Variation in Volunteerism. The Sociological Quarterly, 51(1), 46-64.

Stettes, O. (2013). Verdienstunterschiede - kein Anlass zur Skandalisierung. ifo Schnelldienst, 66(7), 6-9.

Voß, G.-G. (2010). Arbeit als Grundlage menschlicher Existenz: Was ist Arbeit? Zum Problem eines allgemeinen Arbeitsbegriffs. In F. Böhle, G.-G. Voß, \& G. Wachtler (Hg.), Handbuch Arbeitssoziologie (S. 23-80). Wiesbaden: VS Verlag für Sozialwissenschaften.

Wilensky, H. L. (1961). Orderly Careers and Social Participation: The Impact of Work History on Social Integration in the Middle Mass. American Sociological Review, 26(4), 521-539.

Windzio, M. (2013). Regressionsmodelle für Zustände und Ereignisse: Eine Einführung. Wiesbaden: Springer Fachmedien.

Wooldridge, J. M. (2002). Econometric analysis of cross section and panel data. Cambridge, Mass: MIT Press.

Wymer, W. (2011). The Implications of Sex Differences on Volunteer Preferences. Voluntas, 22(4), 831-851.

Zierau, J. (2001). Genderperspektive - Freiwilligenarbeit, ehrenamtliche Tätigkeit und bürgerschaftliches Engagement bei Männern und Frauen. In S. Picot (Hg.), Freiwilliges Engagement in Deutschland. Ergebnisse der Repräsentativerhebung zu Ehrenamt, Freiwilligenarbeit und bürgerschaftlichem Engagement.. Band 3: Frauen und Männer, Jugend, Senioren, Sport (S. 15-110). Stuttgart [u.a.]: Kohlhammer. 


\section{Anhang}

Tabelle 4: Heckprobit-Modell - Erklärungsfaktoren der Leitungspositionen in Ehrenämtern

\begin{tabular}{lcccc}
\hline & \multicolumn{2}{c}{ Hauptgleichung } & \multicolumn{2}{c}{ Auswahlgleichung } \\
\hline Geschlecht (Frau=1) & b & Std. err. & b & Std. err. \\
\hline 14 bis 24 Jahre & $-0,218^{* * *}$ & $(0,035)$ & $-0,115^{* * *}$ & $(0,021)$ \\
\hline 25 bis 34 Jahre & $-0,053$ & $(0,085)$ & $-0,084$ & $(0,051)$ \\
\hline 35 bis 44 Jahre & $-0,125^{*}$ & $(0,060)$ & $-0,172^{* * *}$ & $(0,037)$ \\
\hline 45 bis 54 Jahre & $-0,147^{* *}$ & $(0,051)$ & $-0,024$ & $(0,032)$ \\
\hline 55 bis 64 Jahre & Ref. & Ref. & Ref. & Ref. \\
\hline$>$ 65 Jahre & 0,059 & $(0,053)$ & $-0,049$ & $(0,034)$ \\
\hline Keine Kinder & 0,146 & $(0,079)$ & $-0,261^{* * *}$ & $(0,049)$ \\
\hline Kinder ohne Betreuungsaufwand & Ref. & Ref. & Ref. & Ref. \\
\hline Kinder mit tw. Betreuungsaufwand & 0,004 & $(0,076)$ & $0,119^{*}$ & $(0,051)$ \\
\hline Kinder mit gänzl. Betreuungsaufwand & $-0,163^{*}$ & $(0,065)$ & $0,240^{* * *}$ & $(0,042)$ \\
\hline kein Migrationshintergrund & $-0,119^{*}$ & $(0,058)$ & $0,278^{* * *}$ & $(0,035)$ \\
\hline 1. Migrantengeneration & Ref. & Ref. & Ref. & Ref. \\
\hline 2. Migrantengeneration & $-0,018$ & $(0,099)$ & $-0,439^{* * *}$ & $(0,050)$ \\
\hline erwerbstätig & 0,075 & $(0,056)$ & $-0,177^{* * *}$ & $(0,033)$ \\
\hline arbeitslos & Ref. & Ref. & Ref. & Ref. \\
\hline nicht erwerbstätig & $-0,117$ & $(0,085)$ & $-0,096^{*}$ & $(0,048)$ \\
\hline Rentner & $-0,033$ & $(0,076)$ & $-0,037$ & $(0,046)$ \\
\hline sonstige Erwerbstätige & $-0,132$ & $(0,069)$ & $-0,018$ & $(0,043)$ \\
\hline noch Schüler & $-0,179^{*}$ & $(0,073)$ & 0,060 & $(0,044)$ \\
\hline Hauptschulabschluss & $0,195^{*}$ & $(0,091)$ & $-0,039$ & $(0,054)$ \\
\hline Realschulabschluss & $-0,001$ & $(0,056)$ & $-0,234^{* * *}$ & $(0,031)$ \\
\hline Abitur & Ref. & Ref. & Ref. & Ref. \\
\hline Ostdeutschland & 0,065 & $(0,039)$ & $0,223^{* * *}$ & $(0,023)$ \\
\hline Freizeitbereich & $-0,007$ & $(0,039)$ & $-0,063^{* *}$ & $(0,024)$ \\
\hline sozialer Bereich & Ref. & Ref. & - & - \\
\hline politischer Bereich & 0,031 & $(0,043)$ & - & - \\
\hline religiöser Bereich & 0,018 & $(0,051)$ & - & - \\
\hline technischer Bereich & 0,019 & $(0,081)$ & - & - \\
\hline
\end{tabular}

Fortsetzung Tabelle 4 nächste Seite 
Fortsetzung Tabelle 4

\begin{tabular}{|c|c|c|c|c|}
\hline & \multicolumn{2}{|c|}{ Hauptgleichung } & \multicolumn{2}{|c|}{ Auswahlgleichung } \\
\hline & $\mathbf{b}$ & Std. err. & b & Std. err. \\
\hline Verein & Ref. & Ref. & - & - \\
\hline Partei/Verband & $0,292^{* * *}$ & $(0,057)$ & - & - \\
\hline Kirche & $-0,125$ & $(0,078)$ & - & - \\
\hline Selbsthilfe/Projekt & $-0,293^{* * *}$ & $(0,050)$ & - & - \\
\hline sonstige Einrichtungen & $-0,181^{* * *}$ & $(0,052)$ & - & - \\
\hline Hauptamtliche in Organisation $(\mathrm{ja}=1)$ & $-0,370^{* * *}$ & $(0,036)$ & - & - \\
\hline täglich engagiert & $0,351^{* * *}$ & $(0,079)$ & - & - \\
\hline wöchentlich engagiert & Ref. & Ref. & - & - \\
\hline monatlich engagiert & $-0,189^{* * *}$ & $(0,034)$ & - & - \\
\hline Seltener/nie engagiert & $-0,825^{* * *}$ & $(0,068)$ & - & - \\
\hline aktiv $<3$ Jahre & $-0,155^{* * *}$ & $(0,042)$ & - & - \\
\hline aktiv 3 bis 10 Jahre & Ref. & Ref. & - & - \\
\hline aktiv > 10 Jahre & 0,037 & $(0,037)$ & - & - \\
\hline finanzielle Situation (sehr) gut & - & - & Ref. & Ref. \\
\hline finanzielle Situation befriedigend & - & - & 0,015 & $(0,021)$ \\
\hline finanzielle Situation (sehr) schlecht & - & - & 0,015 & $(0,027)$ \\
\hline Netzwerk klein & - & - & $-0,308^{* * *}$ & $(0,023)$ \\
\hline Netzwerk mittel & - & - & Ref. & Ref. \\
\hline Netzwerk groß & - & - & $0,276^{* * *}$ & $(0,024)$ \\
\hline$<3$ Jahre am Ort & - & - & $-0,134^{* * *}$ & $(0,038)$ \\
\hline 3 bis 10 Jahre am Ort & - & - & $-0,060^{*}$ & $(0,027)$ \\
\hline > 10 Jahre am Ort & - & - & Ref. & Ref. \\
\hline seit der Geburt am Ort & - & - & $-0,025$ & $(0,024)$ \\
\hline nicht religiös & - & - & Ref. & Ref. \\
\hline schwach religiös & - & - & $0,057^{*}$ & $(0,027)$ \\
\hline mittel religiös & - & - & $0,265^{* * *}$ & $(0,025)$ \\
\hline stark religiös & - & - & $0,678^{* * *}$ & $(0,031)$ \\
\hline kein polit./gesell. Interesse & - & - & Ref. & Ref. \\
\hline polit./gesell. Interesse mittel & - & - & $0,220^{* * *}$ & $(0,031)$ \\
\hline polit./gesell. Interesse stark & - & - & $0,444^{* * * *}$ & $(0,033)$ \\
\hline Konstante & $0,518^{* * *}$ & $(0,071)$ & $-0,680^{* * *}$ & $(0,046)$ \\
\hline rho & $-0,456^{* * *}$ & $(0,052)$ & & \\
\hline $\mathrm{N}$ & 19.995 & & & \\
\hline $\mathrm{N}$ (unzensiert) & 6.996 & & & \\
\hline
\end{tabular}

Quelle: Freiwilligensurvey 2009 (eigene Berechnungen) $/{ }^{*} \mathrm{p}<0.05,{ }^{* *} \mathrm{p}<0.01,{ }^{* * *} \mathrm{p}<0.001$ 
Tabelle 5: Kategorisierung der Engagementbereiche im Freiwilligensurvey 2009

\begin{tabular}{|c|c|}
\hline Neue Kategorie & Originalkategorien \\
\hline Freizeitbereich & Sport und Bewegung, Kultur und Musik, Freizeit und Geselligkeit \\
\hline Sozialer Bereich & $\begin{array}{l}\text { Sozialer Bereich, Gesundheitsbereich, Schule oder Kindergarten, } \\
\text { Außerschulische Jugendarbeit, Erwachsenenbildung }\end{array}$ \\
\hline Politischer Bereich & $\begin{array}{l}\text { Politik und politische Interessenvertretung; Umwelt, Naturschutz, } \\
\text { Tierschutz; Berufl. Interessenvertretung außerhalb des Betriebs, } \\
\text { Justiz und Kriminalitätsprobleme, Bürgerschaftl. Engagement am } \\
\text { Wohnort }\end{array}$ \\
\hline Religiöser Bereich & Kirchlicher oder religiöser Bereich \\
\hline Technischer Bereich & Unfall- oder Rettungsdienst, freiwillige Feuerwehr \\
\hline
\end{tabular}

Tabelle 6: Kategorisierung der Organisationsformen im Freiwilligensurvey 2009

\begin{tabular}{ll}
\hline Neue Kategorie & Originalkategorien \\
\hline Verein & Verein \\
\hline Partei/Verband & Partei, Verband, Gewerkschaft \\
\hline Kirche & Kirche oder religiöse Vereinigung \\
Selbsthilfe & $\begin{array}{l}\text { Selbsthilfegruppe, eine Initiative oder ein Projekt, eine selbst orga- } \\
\text { nisierte Gruppe, allein, nicht in einer Gruppe, Organisation oder } \\
\text { Einrichtung }\end{array}$ \\
\hline Sonst. Einrichtung & $\begin{array}{l}\text { Eine statl. Oder kommunale Einrichtung, Eine private Einrichtung, } \\
\text { Stiftung, Sonstiges }\end{array}$ \\
\hline
\end{tabular}




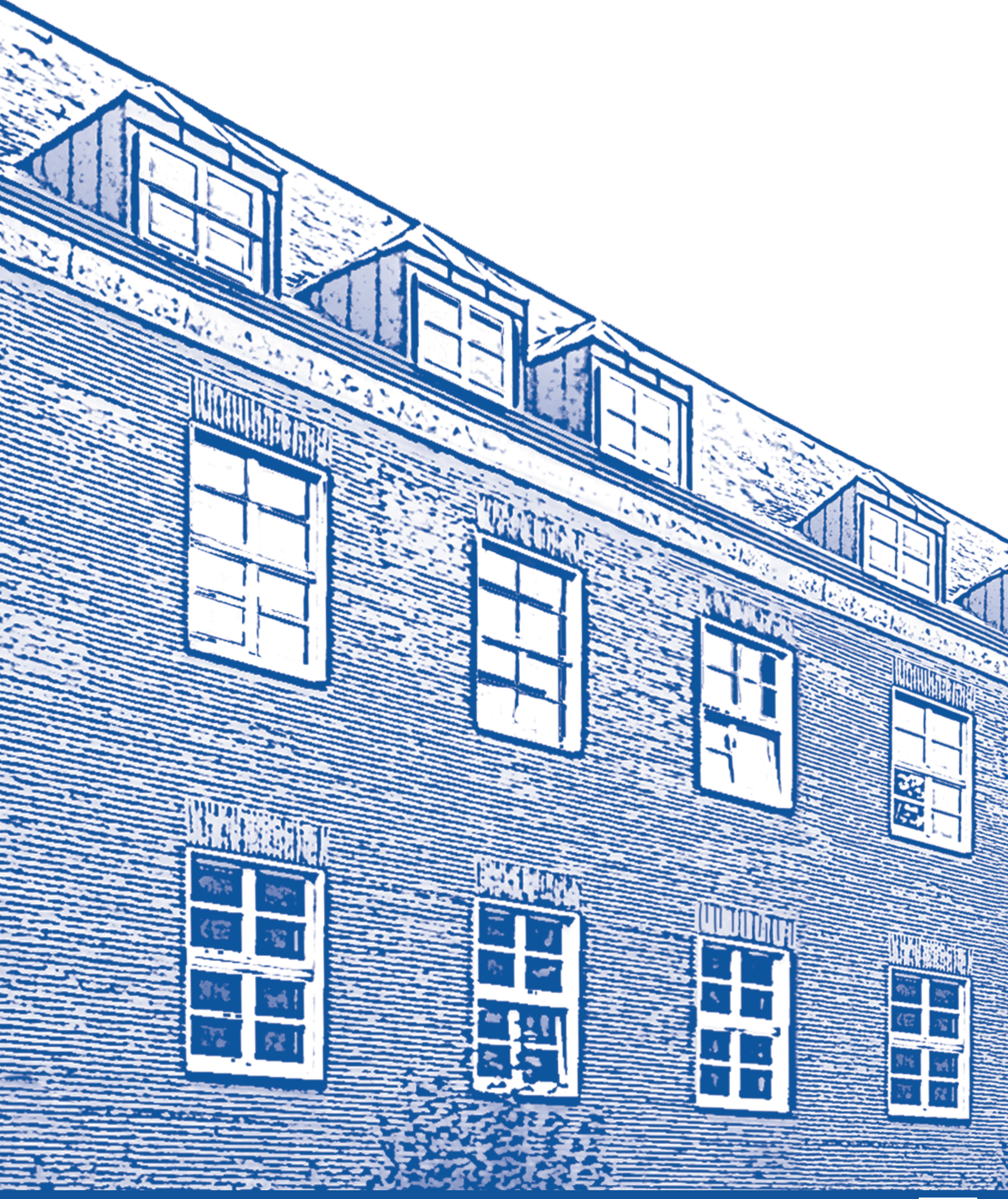

Institut für Soziologie | Universität Duisburg-Essen | Germany IfS 